\title{
Efficient Wavelength Conversion in Silicon Microring CROWs (Project Report 1201308-Y2)
}

\author{
SHAYAN MOOKHERJEA \\ Department of Electrical and Computer Engineering, University of California, San Diego, MC 0407 La \\ Jolla CA 92093-0407 USA \\ Email:smookher@ucsd.edu
}

\section{Introduction and Goals}

The main goal of this NSF-funded project [1201308 - Year 2] is to develop an integrated photonics device technology based on silicon photonics which can be used for compact and efficient nonlinear classical and quantum photonics applications. "Mixers" in the title reflects the use of nonlinear wave mixing, such as through four-wave mixing, which is the foundational optical process for wide-span wavelength conversion and entangled photon-pair generation in silicon photonics.

Potential nonlinear impairments at $1.55 \mu \mathrm{m}$ wavelength in silicon waveguides arise from free-carrier absorption (FCA) losses due to two-photon absorption (TPA) generated carriers (electrons and holes). In our designs, these losses were mitigated in part by reducing the free-carrier lifetime through reverse biased $\mathrm{p}$ i-n diodes fabricated across the waveguide cross-section [1]. Besides waveguides, this project also studied microring resonators and coupledresonator optical waveguides (CROWs) [2], which may be useful for filters [3] and lasers [4], among other devices. Silicon microring CROWs were fabricated and measured [5]. Using these devices, we showed a significant improvement in the conversion efficiency of channelized four-wave mixing (FWM) in the $\mathrm{C}$ band (see Fig. 1).

Some degree of fabrication disorder is inevitable in foundry-fabricated silicon photonics. We considered for adoption and implementation a number of ideas such as post-fabrication trimming using high-resolution experimental techniques which we had previously developed [5],[6]. However, CROWs have a wider passband than a single microring resonator, and it was seen that relatively simple thermal control of the CROW device using a thermo-electric controller attached to the chip mount was adequate for the nonlinear mixing demonstrations performed in this project.

Note that an accurate design of the waveguide-resonator coupling is important and often requires time-consuming simulations, since there can be significant deviations from conventional coupled-mode theory in the high-index-contrast regime of silicon photonics[8]. Thus, a portion of the research activity in this 
project addresses the challenges of designing high-index contrast devices and comparing the results of fabrication to the design intention.

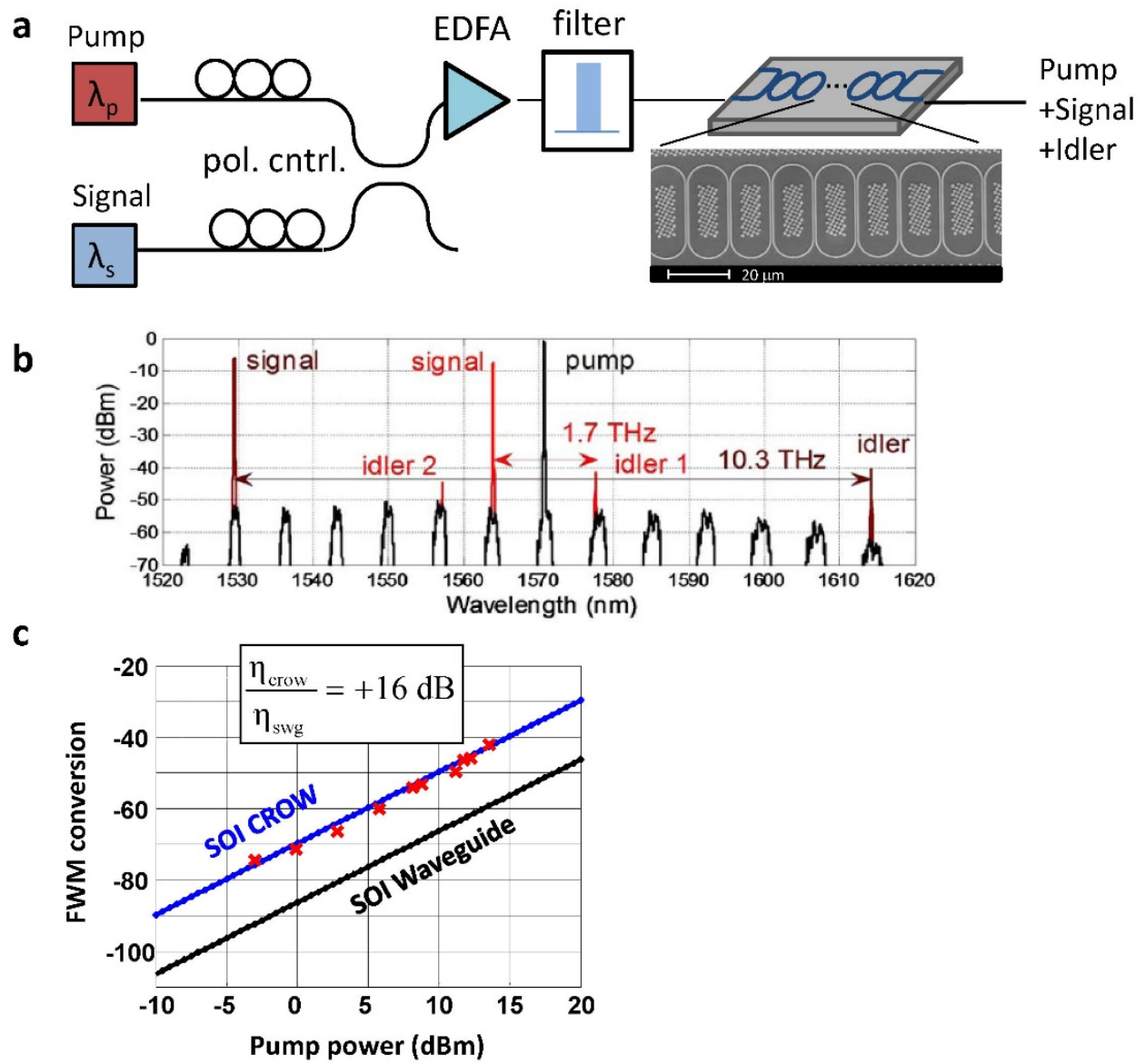

\begin{abstract}
Figure 1(a) Schematic of experimental configuration for measuring wavelength conversion using a coupled-resonator optical waveguide (CROW) consisting of coupled microring resonators in silicon photonics. (b) Measurements of two representative experiments, showing short-range and long-range wavelength conversion, over frequency spans of $1.7 \mathrm{THz}$ and 10.3 $\mathrm{THz}$, respectively. (c) The CROW structure achieves enhanced conversion efficiency over a comparable conventional silicon photonic waveguide by $16 \mathrm{~dB}$.
\end{abstract}

\title{
2. Activities
}

Progress in year 2 (2013-14) builds upon research activities reported during year 1 of this project. Previously, the large dispersion of directional couplers was studied and experimentally verified [9]. An analytical expression was derived for the coupling dispersion to guide the device design in this project [10]. These results were helpful in accurately designing devices in which the measured optical group index was close to the design target (within 5\%) across a large number of microchips.

Nonlinearity can affect the transmission function of racetrack resonators in an interesting way, such as inducing bistability [11]. However, we were able to 
avoid the bistable regime in most of the measurements that we performed even at the elevated power levels used in FWM. Observing the microring resonator with an infrared camera can help in performing the experiments [12], [13].

The CROW devices are designed using racetrack resonators which consist of both straight and curved waveguide sections. We used both passive waveguides and those with a p-i-n diode embedded across the waveguide cross-section for carrier sweepout. The latter type of waveguide was used to design and fabricate microring resonator devices which were later used as sources of entangled photon-pairs generated at telecommunications wavelengths [14].

Compared to conventional waveguides, a CROW provides significant resonant enhancement of the conversion efficiency [15], [16], but also offers a relatively wide passband (up to several nanometers). These are two useful reasons to study CROWs in the context of photonic mixers. However, the ab-initio simulation of such devices is not computationally feasible on conventional PC workstations. Basic principles of the design of such devices can utilize analytical derivations already published and were used as the foundation for our device designs in this project. For example, an analytical treatment of pulse propagation clearly shows the beneficial role played by slow light [17], [18]. However, it should also be recognized that CROWs have a relatively high dispersion even at band-center, which can impact nonlinear conversion efficiency [19]-[21].

\section{$10 \mathrm{Gbit} / \mathrm{s}$ NRZ wavelength conversion with CW pump}

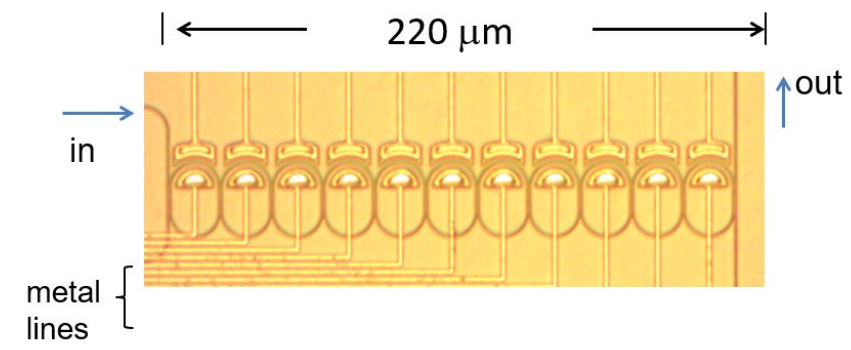

$1548 \mathrm{~nm}$

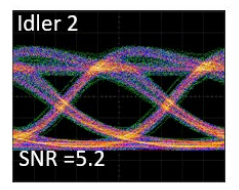

$1556 \mathrm{~nm}$

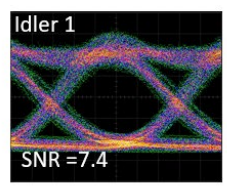

$1563 \mathrm{~nm}$

CW pump
$1571 \mathrm{~nm}$

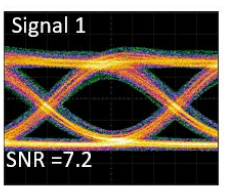

$1578 \mathrm{~nm}$

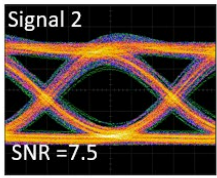

Figure 2 Wavelength conversion of NRZ modulated data using four-wave mixing in a silicon CROW. Inputs were in the L-band and were up-converted to the $\mathrm{C}$ band. 


\section{Progress and Impact}

We have studied wavelength conversion between the C-band and L-band experimentally [22] and demonstrated wavelength up-conversion of NRZ modulated data $(10 \mathrm{Gbit} / \mathrm{s})$ in a "live" wavelength-division multiplexed (WDM) optical network that is part of a campus testbed (see Fig. 2). The device used to perform wavelength conversion was a CROW structure with tunable heaters for adjusting the passband wavelength. This is necessary because the wavelengths in a communication network are fixed. Although the CROW device itself has a very high nonlinear conversion efficiency, the edge couplers to fiber were not optimized, and the measurement of eye diagrams of the converted channels required external amplification using EDFAs to compensate for the losses. Two channels were simultaneously wavelengthconverted from the L-band to the C-band, preserving the data.

This year, research progress also demonstrated improved FWM in carrier-swept silicon waveguides and in single microring resonators in which a $\mathrm{p}-\mathrm{i}-\mathrm{n}$ diode was fabricated across the waveguide cross-section to sweep out electronic free carriers [23]. The silicon waveguides do not have a channelized response like that of the CROWs, and the FWM conversion process can be tuned continuously over a range of wavelengths that is limited by the bandwidth of the phase-matching. In some cases, phase-matching can be achieved in silicon waveguides over very wide spectral ranges [24].

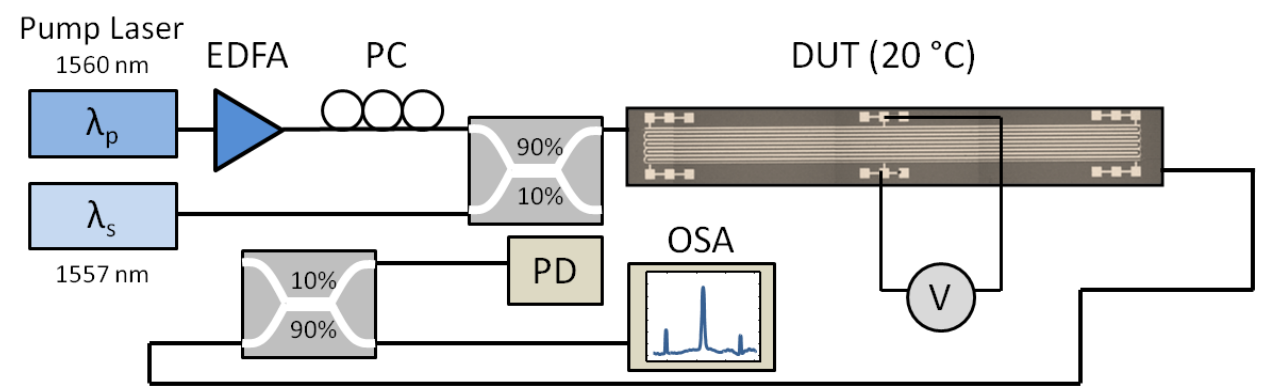

Figure 3 Four-wave mixing measurement setup using $\mathrm{cw}$ pump and signal lasers. The silicon nanophotonic waveguide is $6.35 \mathrm{~cm}$ long, with $\mathrm{p}$ and $\mathrm{n}$ doped regions along its length.

In silicon waveguides that were folded into "paperclip" structures to reduce the on-chip footprint, as shown in Fig. 3, a low propagation loss was measured to be $-0.74 \mathrm{~dB} / \mathrm{cm}$ at $1550 \mathrm{~nm}$ wavelengths. A conversion efficiency of $-8 \mathrm{~dB}$ was measured (defined as idler output power divided by signal input power). Application of reverse bias $(10 \mathrm{~V})$ improved conversion efficiency by nearly 5 $\mathrm{dB}$ compared to the no-bias case. The mitigation of nonlinear loss by applying a reverse-bias voltage to a doped silicon waveguide was previously studied in the context of a silicon Raman laser [1]. 
Nonlinear wavelength conversion overcomes some limitations of other tuning techniques in silicon photonics. All optical tuning using gain has been proposed [25] but is not feasible in silicon photonics due to the lack of optical gain. Compared to slow thermo-optic tuning [26], all-optical wavelength can achieve a much faster response. As shown in Fig. 1, wavelength conversion over more than $10 \mathrm{THz}$ was achieved using FWM. On the other hand, for wavelength conversion over relatively short frequency ranges (less than $100 \mathrm{GHz}$ ), an allelectro-optic approach may be simpler and more convenient. Towards this goal, high-bandwidth integrated electro-optic modulators were demonstrated in subsequent research [27].

The conversion efficiency of CROWs increases with the number of coupled resonators. However, disorder can limit the length of the chain that is achievable without collapse of the passband [28]. Disorder in CROWs can show other complex physical behavior such as localization of light. In contrast to conventional dielectrics, localization can be controlled electronically in silicon structures with embedded dopants [29]. This may lead to new insights into how to harness disorder which cannot be eliminated.

Impact on Human Resources: NSF funds supported two graduate students and one undergraduate student. They worked on device design, fabrication and testing.

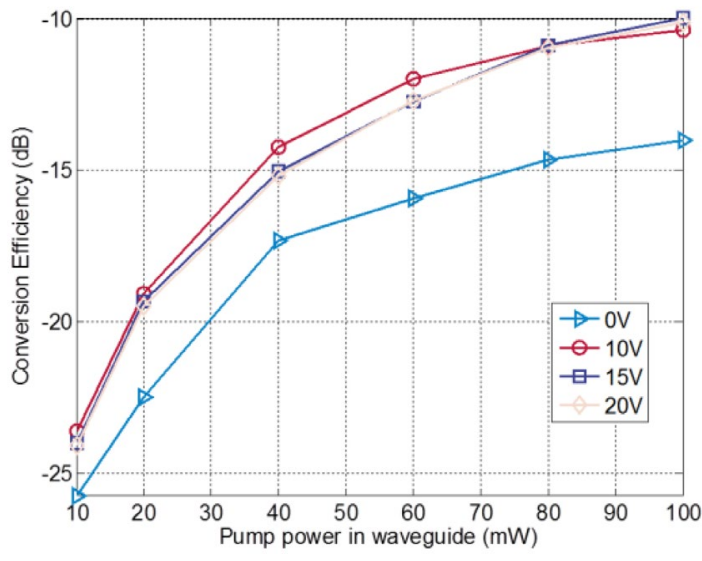

Figure 4 Four-wave mixing conversion efficiency versus $\mathrm{CW}$ pump power (into the waveguide) for different reverse bias voltages.

\section{Subsequent Extensions}

The fact that wavelength conversion efficiency can approach 10\% (see Fig. 4) with only $100 \mathrm{~mW}$ pump power in silicon waveguides is promising for wavelength conversion and all-optical signal processing using conventional milliwatt-scale laser diodes. However, optical microsystems that will implement wavelength conversion in WDM optical networks need many additional components and

system improvements before practical use. Additional work was done in this project to design wavelength selective combiners / splitters that can multiplex and de-multiplex pump wavelengths from signal and idler wavelengths [30]. 
Adding tunable filters and multiplexers may introduce additional optical loss, and therefore, a method of monolithic integration of filters with mixers can be useful.

Quantum light generation on silicon photonic chip is emerging as a topic of current relevance [31]. Research in the first year of this project laid the groundwork for a detailed design and device optimization which eventually led to a significant improvement in the pair-generation properties of silicon microring resonators [32], [33]. Nevertheless, it is also possible to improve the pair-generation properties of devices based on the stronger chi-(2) nonlinearity, such as in periodically-poled thin-film lithium niobate [34]. More research is needed is to address which approach is best suited for practical use.

\section{Open-Access Reporting Initiative}

PRAISE: This open-access document is provided in support of our PRAISE (Public Report of Activities, Impact and Subsequent Extensions) initiative. What is it? An open-access document shared with the public which describes the research outcomes of publicly-funded projects. For us, these projects are typically funded by the NSF (National Science Foundation).

\section{References}

[1] H. Rong et al., “An all-silicon Raman laser,” Nature, vol. 433, no. 7023, pp. 292-294, Jan. 2005, doi: 10.1038/nature03273.

[2] F. Morichetti, C. Ferrari, A. Canciamilla, and A. Melloni, "The first decade of coupled resonator optical waveguides: bringing slow light to applications," Laser \& Photon. Rev., vol. 6, no. 1, pp. 74-96, Jan. 2012, doi: 10.1002/lpor.201100018.

[3] S. Mookherjea, "Spectral characteristics of coupled resonators," J. Opt. Soc. Am. B, vol. 23, no. 6, p. 1137, Jun. 2006, doi: 10.1364/JOSAB.23.001137.

[4] S. Mookherjea, "Semiconductor coupled-resonator optical waveguide laser," Appl. Phys. Lett., vol. 84, no. 17, pp. 3265-3267, Apr. 2004, doi: 10.1063/1.1719278.

[5] M. L. Cooper et al., "235-ring Coupled-Resonator Optical Waveguides," in Conference on Lasers and Electro-Optics 2010, San Jose, California, 2010, p. CTuHH3. doi: 10.1364/CLEO.2010.CTuHH3.

[6] Y. Shen, I. B. Divliansky, D. N. Basov, and S. Mookherjea, "Electricfield-driven nano-oxidation trimming of silicon microrings and interferometers," Opt. Lett., vol. 36, no. 14, p. 2668, Jul. 2011, doi: 10.1364/OL.36.002668. 
[7] Y. Shen, I. B. Divliansky, D. N. Basov, and S. Mookherjea, "Perfect setand-forget alignment of silicon photonic resonators and interferometers," in Optical Fiber Communication Conference/National Fiber Optic Engineers Conference 2011, Los Angeles, California, 2011, p. PDPC3. doi: 10.1364/OFC.2011.PDPC3.

[8] M. L. Cooper and S. Mookherjea, "Numerically-assisted coupled-mode theory for silicon waveguide couplers and arrayed waveguides," Opt. Express, vol. 17, no. 3, p. 1583, Feb. 2009, doi: 10.1364/OE.17.001583.

[9] R. Aguinaldo, Yiran Shen, and S. Mookherjea, "Large Dispersion of Silicon Directional Couplers Obtained via Wideband Microring Parametric Characterization," IEEE Photon. Technol. Lett., vol. 24, no. 14, pp. 1242-1244, Jul. 2012, doi: 10.1109/LPT.2012.2198639.

[10] M. L. Cooper and S. Mookherjea, "Modeling of Multiband Transmission in Long Silicon Coupled-Resonator Optical Waveguides," IEEE Photon. Technol. Lett., vol. 23, no. 13, pp. 872-874, Jul. 2011, doi: 10.1109/LPT.2011.2141657.

[11] S. Mookherjea and M. A. Schneider, "The nonlinear microring adddrop filter," Opt. Express, vol. 16, no. 19, p. 15130, Sep. 2008, doi: 10.1364/OE.16.015130.

[12] M. L. Cooper, G. Gupta, J. S. Park, M. A. Schneider, I. B. Divliansky, and S. Mookherjea, "Quantitative infrared imaging of silicon-on-insulator microring resonators," Opt. Lett., vol. 35, no. 5, p. 784, Mar. 2010, doi: 10.1364/OL.35.000784.

[13] S. Mookherjea and H. R. Grant, "High dynamic range microscope infrared imaging of silicon nanophotonic devices," Opt. Lett., vol. 37, no. 22, p. 4705, Nov. 2012, doi: 10.1364/OL.37.004705.

[14] M. Savanier, R. Kumar, and S. Mookherjea, "Optimizing photon-pair generation electronically using a $p-i-n$ diode incorporated in a silicon microring resonator," Appl. Phys. Lett., vol. 107, no. 13, p. 131101, Sep. 2015, doi: 10.1063/1.4932047.

[15] S. Mookherjea and A. Yariv, "Second-harmonic generation with pulses in a coupled-resonator optical waveguide," Phys. Rev. E, vol. 65, no. 2, p. 026607, Jan. 2002, doi: 10.1103/PhysRevE.65.026607.

[16] S. Mookherjea and A. Yariv, "Kerr-stabilized super-resonant modes in coupled-resonator optical waveguides," Phys. Rev. E, vol. 66, no. 4, p. 046610, Oct. 2002, doi: 10.1103/PhysRevE.66.046610.

[17] S. Mookherjea and A. Yariv, "Optical pulse propagation in the tightbinding approximation," Opt. Express, vol. 9, no. 2, p. 91, Jul. 2001, doi: 10.1364/OE.9.000091.

[18] S. Mookherjea and A. Yariv, "Optical pulse propagation and holographic storage in a coupled-resonator optical waveguide," Phys. Rev. $E$, vol. 64 , no. 6, p. 066602 , Nov. 2001, doi: 10.1103/PhysRevE.64.066602. 
[19] S. Mookherjea and A. Yariv, "Pulse propagation in a coupled resonator optical waveguide to all orders of dispersion," Phys. Rev. E, vol. 65, no. 5, p. 056601, Apr. 2002, doi: 10.1103/PhysRevE.65.056601.

[20] M. L. Cooper et al., "Waveguide dispersion effects in silicon-oninsulator coupled-resonator optical waveguides," Opt. Lett., vol. 35, no. 18, p. 3030, Sep. 2010, doi: 10.1364/OL.35.003030.

[21] S. Mookherjea, D. S. Cohen, and A. Yariv, "Nonlinear dispersion in a coupled-resonator optical waveguide," Opt. Lett., vol. 27, no. 11, p. 933, Jun. 2002, doi: 10.1364/OL.27.000933.

[22] J. R. Ong et al., "Low-power continuous-wave four-wave mixing in silicon coupled-resonator optical waveguides," Opt. Lett., vol. 36, no. 15, pp. 2964-2966, 2011.

[23] J. R. Ong, R. Kumar, R. Aguinaldo, and S. Mookherjea, "Efficient CW Four-Wave Mixing in Silicon-on-Insulator Micro-Rings With Active Carrier Removal," IEEE Photon. Technol. Lett., vol. 25, no. 17, pp. 16991702, Sep. 2013, doi: 10.1109/LPT.2013.2272521.

[24] S. Zlatanovic et al., "Mid-infrared wavelength conversion in silicon waveguides using ultracompact telecom-band-derived pump source," Nature Photon, vol. 4, no. 8, pp. 561-564, Aug. 2010, doi: 10.1038/nphoton.2010.117.

[25] S. Mookherjea, "Using gain to tune the dispersion relation of coupledresonator optical waveguides," IEEE Photon. Technol. Lett., vol. 18, no. 5, pp. 715-717, Mar. 2006, doi: 10.1109/LPT.2006.871144.

[26] R. Aguinaldo et al., "Wideband silicon-photonic thermo-optic switch in a wavelength-division multiplexed ring network," Opt. Express, vol. 22, no. 7, p. 8205, Apr. 2014, doi: 10.1364/OE.22.008205.

[27] X. Wang, P. O. Weigel, J. Zhao, M. Ruesing, and S. Mookherjea, "Achieving beyond-100-GHz large-signal modulation bandwidth in hybrid silicon photonics Mach Zehnder modulators using thin film lithium niobate," APL Photonics, vol. 4, no. 9, p. 096101, Sep. 2019, doi: 10.1063/1.5115243.

[28] S. Mookherjea and M. A. Schneider, "Avoiding bandwidth collapse in long chains of coupled optical microresonators," Opt. Lett., vol. 36, no. 23, p. 4557, Dec. 2011, doi: 10.1364/OL.36.004557.

[29] S. Mookherjea, J. R. Ong, X. Luo, and L. Guo-Qiang, "Electronic control of optical Anderson localization modes," Nature Nanotech, vol. 9, no. 5, pp. 365-371, May 2014, doi: 10.1038/nnano.2014.53.

[30] J. R. Ong, R. Kumar, and S. Mookherjea, "Silicon microring-based wavelength converter with integrated pump and signal suppression," Opt. Lett., vol. 39, no. 15, p. 4439, Aug. 2014, doi: 10.1364/OL.39.004439.

[31] J. R. Ong and S. Mookherjea, "Quantum light generation on a silicon chip using waveguides and resonators," Opt. Express, vol. 21, no. 4, p. 5171, Feb. 2013, doi: 10.1364/OE.21.005171. 
[32] C. Ma, X. Wang, V. Anant, A. D. Beyer, M. D. Shaw, and S. Mookherjea, "Silicon photonic entangled photon-pair and heralded single photon generation with CAR $>12,000$ and $\mathrm{g}^{\wedge}(2)(0)<0006$," Opt. Express, vol. 25, no. 26, p. 32995, Dec. 2017, doi: 10.1364/OE.25.032995.

[33] R. Kumar, M. Savanier, J. R. Ong, and S. Mookherjea, "Entanglement measurement of a coupled silicon microring photon pair source," Opt. Express, vol. 23, no. 15, p. 19318, Jul. 2015, doi: 10.1364/OE.23.019318.

[34] J. Zhao, C. Ma, M. Rüsing, and S. Mookherjea, "High Quality Entangled Photon Pair Generation in Periodically Poled Thin-Film Lithium Niobate Waveguides," Phys. Rev. Lett., vol. 124, no. 16, p. 163603, Apr. 2020, doi: 10.1103/PhysRevLett.124.163603. 